\author{
Oren Y. Kanner ${ }^{1}$ \\ e-mail: oren.kanner@yale.edu \\ Aaron M. Dollar \\ Assistant Professor \\ Mem. ASME \\ e-mail: aaron.dollar@yale.edu \\ Department of Mechanical Engineering, \\ Yale University, \\ New Haven, CT 06511
}

\section{Kinematic Design of an Underactuated Robot Leg for Passive Terrain Adaptability and Stability}

This paper investigates how the passive adaptability of an underactuated robot leg to uneven terrain is affected by variations in design parameters. In particular, the joint torque coupling ratio, segment length ratio, and rest angles are varied to determine configurations that allow for maximum terrain roughness adaptability while minimizing the transmission of disturbance forces to the body. In addition, a series of alternate leg actuation configurations are considered. The results show that a proximal/distal joint torque coupling ratio of 2 with an inverted distal joint, a proximal/distal leg length ratio of 1.25 , and an initial proximal joint angle of $-53 \mathrm{deg}$ maximize the terrain variability over which the robot can remain stable by exerting a near-constant vertical reaction force while minimizing lateral force and moment disturbances. In addition, the spring stiffness ratio allows for a tradeoff to be made between the different performance metrics. Finally, the robot's stability with respect to its posture is discussed. [DOI: 10.1115/1.4024238]

Keywords: underactuated robots, mechanism design of mobile robots, multilegged robots

\section{Introduction}

Good performance over rough terrain is a key factor for the successful implementation of many legged robotic systems. Besides bipeds, work in this area has focused on legged systems that fall into two main groups: (1) more complex systems that close the loop around a variety of sensors in order to control leg placement, stiffness, or force (e.g., Refs. [1-3]) and (2) simpler systems intended to run mostly open-loop, many of which utilize compliance in the legs and/or body (e.g., Refs. [4-7]). While there are clear advantages and shortcomings to both approaches, a simpler system that has excellent open-loop mechanical performance without the need for low-level control (and the sensing and computing that goes with it) for leg placement and stability would be ideal.

In this paper, we begin to investigate the development of legged robots that utilize adaptive underactuation, through differential transmission designs, to allow the legs to naturally adapt to rough terrain to an extent that mechanical compliance alone typically cannot enable. These types of mechanisms have been used extensively in robotic hands in order to enable passive adaptation to a wide range of object sizes, shapes, and positions/orientations in a purely open-loop manner (e.g., Refs. [8-11]). However, as far as the authors are aware, they have yet to be applied to legged robotic systems. For legs with compliance but without differential-like underactuated mechanisms, adaptability is limited, as any change in ground height will result in a change in required force to achieve it (proportional to the spring stiffness). Therefore, when terrain variability is encountered, the forces on the robot (primarily the weight) must redistribute between legs, which will typically result in a net displacement of the robot body

\footnotetext{
Corresponding author

Contributed by the Mechanisms and Robotics Committee of ASME for publication in the Journal of Mechanisms and RoBotics. Manuscript received August 9, 2012; final manuscript received January 25, 2013; published online June 24, 2013. Assoc. Editor: Philippe Wenger.
}

(and certainly a displacement of the base of each leg with a new force on it). However, underactuated mechanisms are differentials that, if properly designed, can allow the legs of the robot to reconfigure irrespective of force. Therefore, it is possible to design a leg such that a wide range of ground heights can be traversed with negligible vertical displacement of the body of the robot (or disturbances forces to it).

In this initial study, we examine how variations in the mechanical parameters of a two-link leg with rotational joints affect its stance adaptability to rough terrain. In particular, we investigate how changes in joint angle, link length, and joint torque coupling ratio affect the ability of the leg to exert a near-constant vertical force on the ground (equal to a $1 / n$ portion of the body weight for a $2 n$-legged robot), with minimal horizontal force and moment disturbances, as determined to be the most applicable to terrain adaptation. These are studied across a wide range of ground heights (and therefore foot placements) in order to find the leg configurations that adapt to the widest range of terrain roughness without actively sensing and modulating the actuator force on the leg-providing the best open-loop passive terrain adaptability while adding minimal disturbance forces to the body of the robot. This is an expanded version of a paper presented at the 2012 ASME Mechanisms and Robotics Conference [12], where we examined only a small subset of actuation schemes and did not address overall robot stability.

We begin this paper by presenting our simplified underactuated leg model and a description of terms. We then evaluate a number of leg classes on the basis of their foot trajectories, eliminating those that would be of little utility. We then describe our simulation, including those parameters that are most relevant and performance metrics used to evaluate the parameter space, and then show how performance with respect to those metrics varies across the space. Finally, we discuss the implications of these results as they apply to the design of passively adaptive underactuated legged robots. 


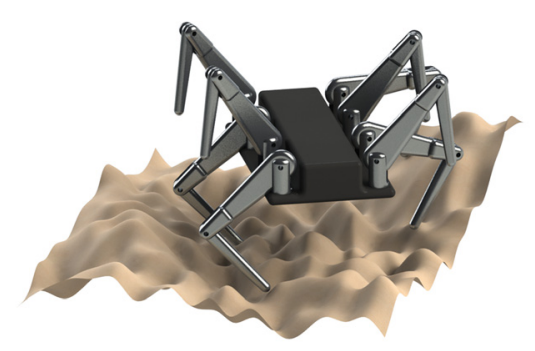

(a)

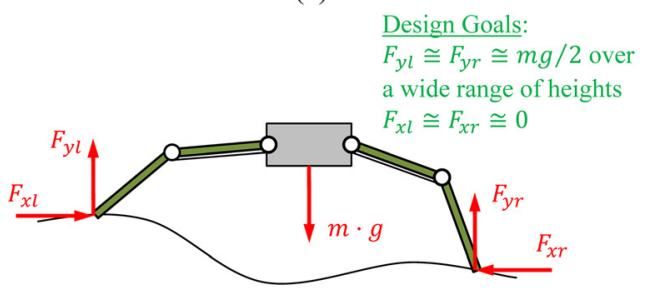

(b)

Fig. 1 (a) Basic robot concept showing alternating tetrapod gait and $(b)$ free-body diagram of an adaptive planar robot standing on uneven terrain. The underactuated leg mechanism allows the robot to be stable across a wide range of ground heights without the need to control the leg force directly.

\section{General Robot Concept}

We envision an eight-legged robot mechanism, with an alternating tetrapod gait (underactuated mechanisms work best in powers of two), that is statically stable at all times (Fig. 1(a)). Each tetrapod group will be connected to a single actuator via a differential transmission that will ensure that the force is equally distributed to each leg without bias to the leg position. Additionally, the contact forces generated at each foot are minimal until all feet of the tetrapod are on the ground (minimizing disturbance during leg placement) as the differential mechanism ensures that the actuator effort first goes to articulation of the compliant joints until they are made effectively rigid by contact (similar to the mechanism described in Ref. [10]). The actuation process is illustrated in Fig. 2. Note that in this paper, we are only addressing the stance behavior of the robot, and in particular, the design of the planar underactuated leg in order to maximize the adaptability to very rough terrain while minimizing disturbance forces on the robot body. The forward motion and steering of the robot will be addressed in future work, and our design concept largely decouples those from the stance behavior.

In order to maximize adaptability of the robot to rough terrain while keeping the body statically stable, we would like to find a leg design that has the following properties: (1) can reach a wide range of ground heights; (2) for a fixed actuator effort (for open-loop operation), the vertical component of the contact force on the ground is nearly constant for a wide range of foot placement locations (e.g., ground heights) and equal to $1 / n$ of the mass (where $n$ is half of the number of legs); (3) the generated horizontal force is close to zero across the range of ground heights to prevent slippage and an unbalanced lateral force; and (4) the net moment on the body is zero. These last three conditions are summarized in Fig. 1(b).

In order to provide insight in the presence of such a large parameter space, we must make a few simplifying assumptions. We assume that the robot's legs will be much lighter than its body, making their inertia during the swing phase very small (and negligible for the purposes of design optimization). Along these lines, the goal of this work is to provide a good basis for motivating the design of legs for this type of system and not to create a detailed dynamic model. We assume that the leg is rigid during contact (the tendon is in parallel with the joint compliance and makes it so) such that the ground contact forces are transmitted directly to the proximal joint (see

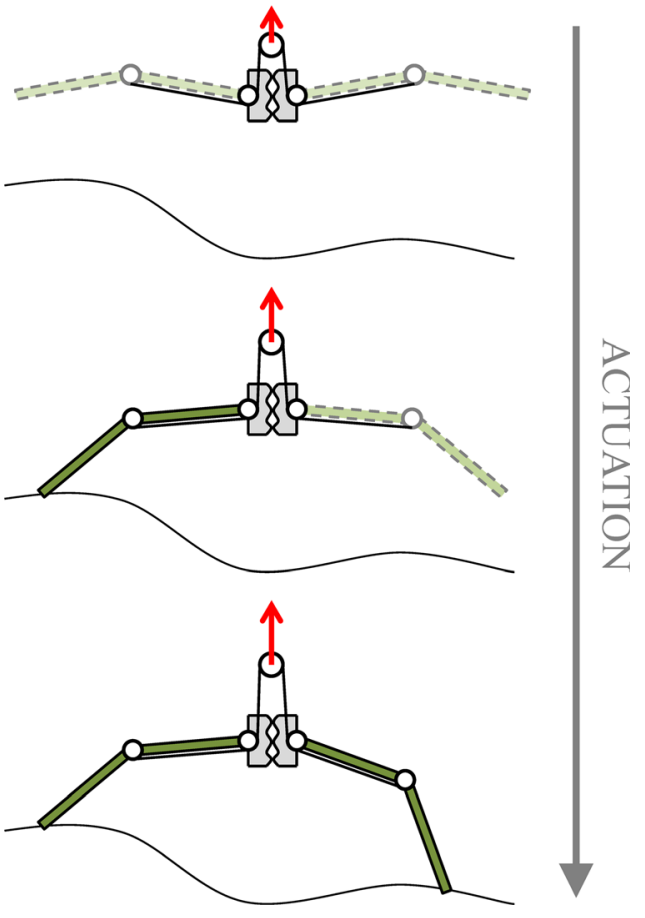

Fig. 2 Diagram of coupled leg-pair actuation process

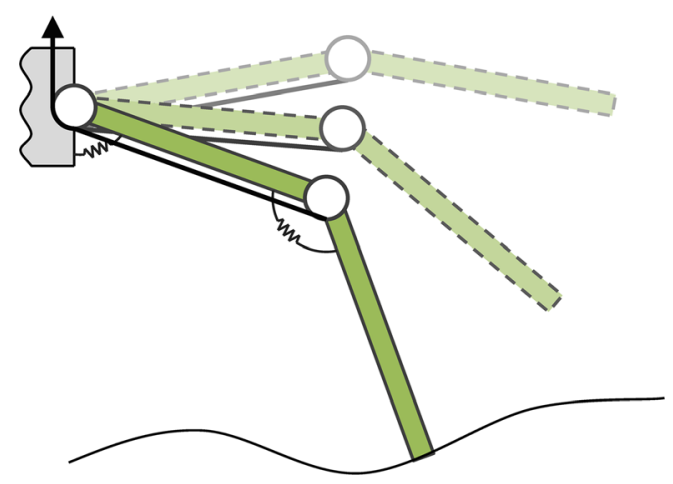

Fig. 3 Diagram of a 2-DOF underactuated leg showing its actuation motion. Note the single tendon driving both joints, which is rigidly attached to the distal joint pulley and couples both joints with a fixed torque ratio.

Eq. (4) below), so that our overall robot free-body diagram can be described as in Fig. 1(b). Also, we assume that the friction between the leg and ground is large enough to prevent slippage. With this broader picture in mind, we will now present our simplified leg model used to evaluate the design space of this robot.

\section{Underactuated Leg Model}

3.1 Basic Leg Description. This model deals with a single underactuated revolute leg. In contrast to prismatic joints, revolute joints are easily driven and well understood and allow for a more complex trajectory space. While prismatic legs might achieve good force performance, their trajectories would have to be largely vertical, requiring a wide body frame for a stable stance. Revolute legs can provide a more sprawled posture while maintaining the required forces for stable walking.

Figure 3 shows the actuation motion of a generic two-link underactuated robot leg as described above. Each joint incorporates a spring to return the leg to some default position in the absence of tendon tension, with the actuation of the leg being used 


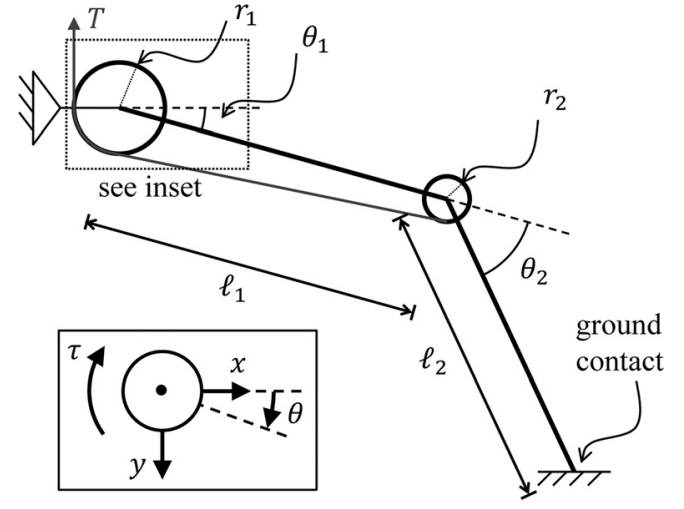

Fig. 4 Representative 2-DOF underactuated robot leg with parameters labeled. Note the inset coordinate frame illustration and definition of $\theta_{2}$ relative to the proximal link.

to lower it to the ground and support the robot's weight. The tendon is rigidly attached to the pulley at the distal joint, which is rigidly attached to the distal link. The pulley at the proximal joint is free-spinning, and we assume frictionless bearings throughout the leg. We also assume that the leg is mass-less, and therefore we ignore inertial effects throughout this analysis.

We compute the torque at each joint as the tendon tension multiplied by the radius of the pulley at the joint or $\tau_{i}=T r_{i}$, where $\tau_{i}$ is the torque in joint $i, T$ is the tendon tension, and $r_{i}$ is the effective pulley radius of joint $i$. When the leg is not in contact with the ground, an increase in the joint torque corresponds to an increase in the joint angle or $\Delta \theta_{i}=\tau_{i} / k_{i}$, where $\Delta \theta_{i}=\theta_{i}-\theta_{i, 0}$ is the joint travel from rest and $k_{i}$ is the spring constant of joint $i$. The initial joint angles $\theta_{i, 0}$ define the rest configuration of the leg. Figure 4 shows a complete leg with parameters labeled and the general coordinate frame used in the model; the origin is at the proximal joint of the leg with positive angles denoting a leg position below the $x$ axis and positive torques following the angle coordinate convention.

3.2 Reaction Forces. In order to evaluate the performance of a given leg design, we need to know the reaction forces at its foot for a given leg configuration and ground height. The following model assumes that the robot body is fixed in space and calculates the reaction force required for the leg to be in static equilibrium. As we will discuss later, it is useful to decompose the ground reaction forces into vertical and horizontal components. Thus, it should be kept in mind that our goal is the evaluation of the two reaction force components, $F_{x}$ and $F_{y}$.

We begin with an underactuated leg in contact with the ground in some configuration. We use the ground height and the leg parameters to determine that configuration, incrementing the tension $T$ in the leg and therefore increasing the joint angles $\theta_{i}=\theta_{i, 0}+T \cdot r_{i} / k_{i}$ until the foot reaches the ground. The position of the foot can easily be evaluated as follows:

$$
\begin{gathered}
x=\ell_{1} \cos \left(\theta_{1}\right)+\ell_{2} \cos \left(\theta_{1}+\theta_{2}\right) \\
y=\ell_{1} \sin \left(\theta_{1}\right)+\ell_{2} \sin \left(\theta_{1}+\theta_{2}\right)
\end{gathered}
$$

Given the configuration of the leg at contact, we treat it as rigid since we assume both ends are fixed in space and determine the ground reaction force generated by the leg. The tendon tension is set to a fixed amount, and we use the sum of moments about each joint to solve for the reaction forces. Looking at the distal segment, we can express that sum of moments as follows:

$$
\tau_{2 a}-\ell_{2}\left[F_{x} \sin \left(\theta_{1}+\theta_{2}\right)+F_{y} \cos \left(\theta_{1}+\theta_{2}\right)\right]=0
$$

where $\tau_{2 a}=T_{2}-k_{2} \Delta \theta_{2}$ is the net torque at the distal joint. Treating the entire leg as a rigid object and the distal joint torque as purely internal, we express the sum of moments at the proximal joint as follows:

$$
\tau_{1 a}-F_{x} \cdot y-F_{y} \cdot x=0
$$

where $\tau_{1 a}=\operatorname{Tr}_{1}-k_{1} \Delta \theta_{1}$ is the net torque at the proximal joint. We now have two linear equations that we can solve for $F_{x}$ and $F_{y}$ to get

$$
\begin{aligned}
& F_{x}=\frac{\tau_{2 a} \cdot x-\tau_{1 a} \ell_{2} \cos \left(\theta_{1}+\theta_{2}\right)}{\ell_{2} \sin \left(\theta_{1}+\theta_{2}\right) \cdot x-\ell_{2} \cos \left(\theta_{1}+\theta_{2}\right) \cdot y} \\
& F_{y}=\frac{\tau_{1 a} \ell_{2} \sin \left(\theta_{1}+\theta_{2}\right)-\tau_{2 a} \cdot y}{\ell_{2} \sin \left(\theta_{1}+\theta_{2}\right) \cdot x-\ell_{2} \cos \left(\theta_{1}+\theta_{2}\right) \cdot y}
\end{aligned}
$$

Equations (1) and (2) allow us to generate the unconstrained motion of the leg as it lowers to the ground, while Eqs. (5) and (6) allow us to calculate the ground reaction forces for a leg given its configuration and final tendon tension. For a given set of leg design parameters, Eqs. (1) and (2) were used to calculate the motion of the leg as the tendon tension was increased. The leg configuration was recorded at a series of equally spaced ground heights until the foot reached its maximum vertical displacement, considered to be the end of the useful trajectory of the leg. For each recorded configuration, Eqs. (5) and (6) were then used to evaluate the reaction forces. These data served allowed us to investigate the effect of leg design on leg performance.

\section{Trajectory Analysis}

4.1 Normalized Parameters/Assumptions. Before looking at the performance of specific leg designs, we reduced our parameter space by performing a qualitative analysis of several classes of leg designs. To simplify our analysis, we reduced a given leg design into two nondimensional parameters and a single intial joint angle term. These nondimensional parameters allow us to ignore the absolute design parameters and simply focus on the general form of the leg. The first parameter was the segment length ratio, defined as $R_{\mathrm{L}}=\ell_{1} / \ell_{2}$. The second parameter was the joint torque coupling ratio, defined as $R_{\mathrm{T}}=\tau_{1} / \tau_{2}$. The initial proximal joint angle, $\theta_{1,0}$, was used as the final design parameter. In order to eliminate the initial distal joint angle as an independent parameter, we constrained the rest configuration of the leg such that the foot always starts at some specific position. The foot was assigned one of two constraints-either $y=0$ (foot at proximal joint height) or $x=0$ (foot below proximal joint). The former required that $\theta_{2,0}=-\sin ^{-1}\left(R_{\mathrm{L}} \sin \left(\theta_{1,0}\right)\right)-\theta_{1,0}$, while the latter required that $\theta_{2,0}=\cos ^{-1}\left(-R_{\mathrm{L}} \cos \left(\theta_{1,0}\right)\right)-\theta_{1,0}$.

The actual dimensions of the leg were normalized for simplicity. Each segment length was normalized by some nominal length and the total leg length was constrained to a normalized value of 2 (such that with $R_{\mathrm{L}}=1$ we would have $\ell_{1}=\ell_{2}=1$ ). The joint pulley radii were similarly normalized by the nominal segment length, as were the joint spring constants. In addition, all the forces were normalized by the final tendon tension.

Rather than including the spring constants as separate design parameters, they were assumed to have a fixed ratio, defined as $R_{\mathrm{k}}=k_{1} / k_{2}$, and set to small values, such that the spring torque values at maximal extension would be at least an order of magnitude smaller than the joint torque. While the spring constants determine the ratio between proximal and distal joint angles, they only exist to return the leg to its initial configuration, and their effect on the forces exerted by the leg was expected to be negligible. We will return to the spring constant ratio later in our discussion.

One important assumption made in the analysis was that the proximal joint of the leg was pinned in space and that the leg would not reconfigure once it made contact with the ground. In the absence of a large horizontal reaction force, one that would 
overcome static friction, the latter assumption should always be valid as the leg would be in equilibrium. One final point to note is that the parameters were evaluated only within physically realistic bounds. The lower and upper bounds of the kinematic parameters (e.g., pulley radii and leg lengths) were chosen such that a robot could reasonably be constructed with those specifications and any designs with interfering parts were ignored.

With all of these assumptions in mind, we categorized leg designs according to the following scheme. First, a series of designs were chosen where the initial foot position was constrained to $y=0$. Within this group, legs were split by the direction of each actuator, where $\tau_{1}$ and $\tau_{2}$ could be either positive or negative, and by the initial configuration, where $\theta_{1,0}$ could be either positive or negative (knee-up or knee-down). Finally, the group of legs for which the initial foot position was constrained to $x=0$ was considered.

4.2 Trajectory Quality. When evaluating legs qualitatively, we must define what makes a "good" leg. One requirement is that the foot moves downward as tendon tension increases; otherwise, the leg cannot reach the ground and is useless by definition. Another qualification is a leg whose configuration is never singular. A singular configuration is one where subsequent behavior of the mechanism is unpredictable or where the forces involved become infinite or nondeterministic. In this case, if we look at our force definitions above (Eqs. (5) and (6)), we can reduce the denominator of both expressions to $L_{1} L_{2} \sin \left(\theta_{2}\right)$. Therefore, if $\theta_{2}$ is equal to $0 \mathrm{deg}$ or $180 \mathrm{deg}$, then the reaction forces required to balance the leg are indeterminate. Finally, the reaction force required for equilibrium must oppose the robot's weight; if this is not the case, the leg would simply collapse. With these basic trajectory characteristics in mind, we will begin by looking at the legs that start with the foot at $y=0$.

4.3 Positive Joint Torque Ratio. Legs that fall into this category have both joints moving in the same direction. The group of legs where both joints move in the negative direction can be excluded immediately since the foot will always move up as tendon tension is increased. Figure 5 shows the remaining two cases where both joint torques are positive and the initial joint angles vary. The leg in Fig. 5(a) meets all of the characteristics for a good trajectory - its foot always moves down with increasing tendon tension, its configuration is never singular, and the ground reaction force will always oppose the robot's weight. The leg in Fig. $5(b)$, on the other hand, fails with respect to the singular configuration requirement-most configurations with both joint torques positive and $\theta_{1,0}>0$ will pass through a singular configuration. Additionally, in the rest configuration the distal joint will be below the foot, making it likely that it will make contact before the foot which is highly undesirable.

4.4 Negative Joint Torque Ratio. Legs that fall into this category have joints that move in opposite directions. Since we can either have either joint be positive or negative, we will deal with each pairing separately. Figure 6 shows the two categories of legs that have $\tau_{1}<0$. First looking at Fig. $6(b)$, it is clear that this class of leg designs will all pass through the singular configuration. Looking at Fig. 6(a), we see a leg that at first glance seems to pass all of our tests for a "good" trajectory; it does not pass through the singular configuration, seems as though it could support the robot's weight, and moves down as tendon tension increases. However, due to the geometry of the leg, the foot will often immediately travel up with increasing tendon tension, not down. Moreover, those legs that move down have a very limited reach, making them undesirable.

Figure 7 shows the two categories of legs that have $\tau_{2}<0$. The leg category shown in Fig. 7(b) fails our last trajectory criterion, in that the ground reaction forces do not oppose the robot's weight, and therefore this type of leg would collapse under the

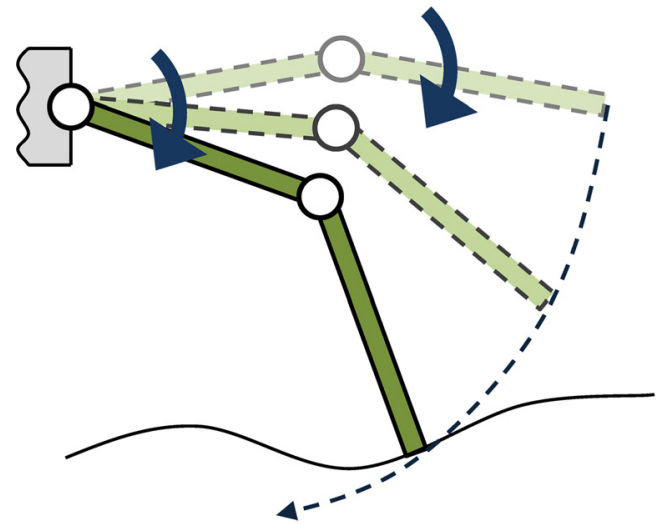

(a)

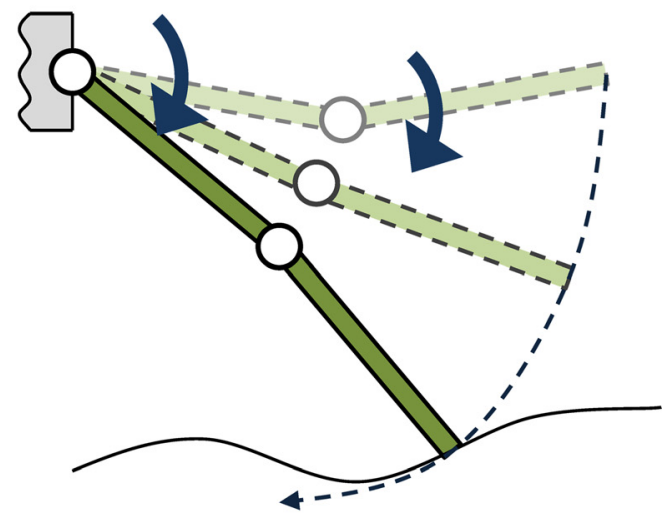

(b)

Fig. 5 Representative robot legs with $\tau_{1}>0$ and $\tau_{2}>0$ and either (a) $\theta_{1,0}<0$ or (b) $\theta_{1,0}>0$

robot's weight given weak springs. Looking at Fig. 7(a), we see that legs of this type will only sometimes pass through a singular configuration. If $\left|R_{\mathrm{T}}\right|$ is much greater than 1 , then the proximal joint will actuate much more quickly than the distal joint, to the point where the foot will begin to move up before $\theta_{2}=0$. Since we cannot conclusively eliminate this specific group of legs based on their trajectories, we will include it in our force analysis later on.

4.5 "Push-Up" Leg. The final class of legs that we will evaluate is what we will call "push-up" legs, or legs where the foot is initially constrained to be located below the proximal joint, as shown in Fig. 8. The basic definition of such a leg is simply that $\theta_{1,0}$ is greater than zero, $\theta_{2,0}$ is defined to put the foot at $x=0$ (see above), and that $\tau_{1}>0$ and $\tau_{2}<0$; otherwise, the foot either moves up or under the robot body. In addition, if $R_{\mathrm{L}}$ is greater than one (i.e., proximal segment longer than distal segment), then there may be no geometrically valid configuration such that the foot is below the proximal joint, with the limit on $R_{\mathrm{L}}$ being dependent on $\theta_{1,0}$. Finally, as the leg actuates, it is effectively moving toward the singular configuration, leading to highly variable forces and poor performance overall.

\section{Performance Analysis and Results}

5.1 Leg Performance Metrics. The overall force performance of an underactuated leg in this analysis was determined by three metrics. The first metric was the effective reach of the leg, or the lowest ground height the leg was capable of reaching $\left(y_{\max }\right)$. This is important since the total length of the leg was 


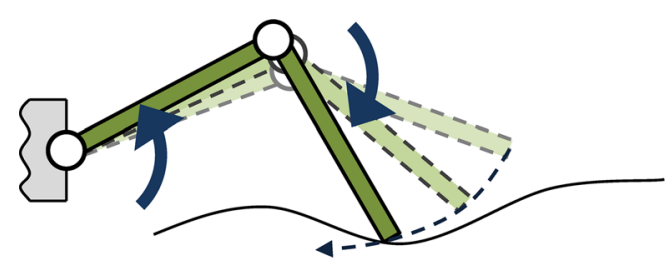

(a)

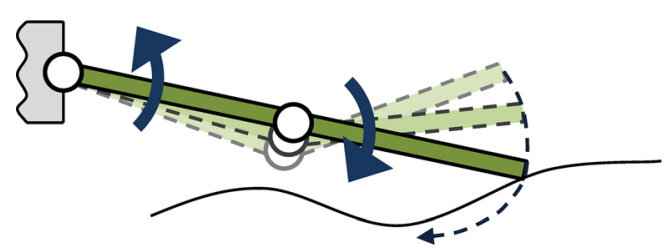

(b)

Fig. 6 Representative robot legs with $\tau_{1}<0$ and $\tau_{3}>0$ and either (a) $\theta_{1,0}<0$ or (b) $\theta_{1,0}>0$

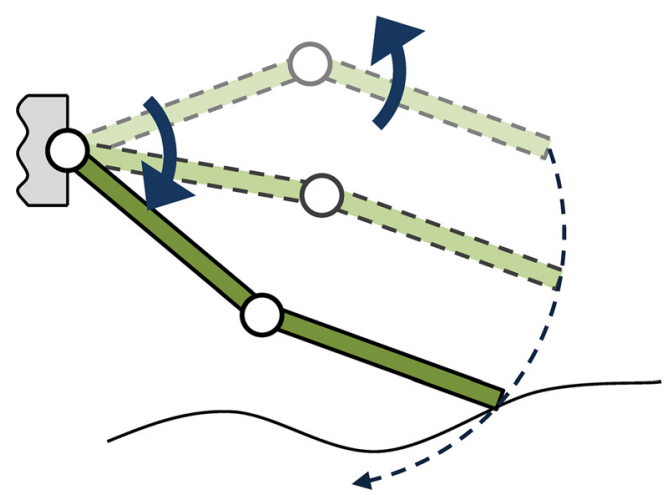

(a)

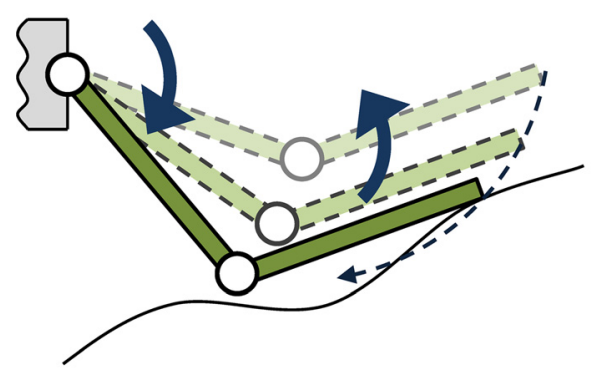

(b)

Fig. 7 Representative robot legs with $\tau_{1}>0$ and $\tau_{2}<0$ and either (a) $\theta_{1,0}<0$ or $(b) \theta_{1,0}>0$

constant across all of the simulations, so it can be viewed as the portion of the leg's length that can be used to reach the ground.

The next two metrics were related to the ground reaction forces exerted by the leg. As described previously, the reaction force was decomposed into its vertical $\left(F_{y}\right)$ and horizontal $\left(F_{x}\right)$ components under the assumption that the global coordinate frame was aligned with the direction of gravity. In this case, $F_{y}$ would be opposed by the robot's weight (or some fraction depending on the number of legs), while $F_{x}$ would be opposed by the frictional force between the foot and the ground.

In our model, the reaction forces are determined to be the set of forces that will ensure that the robot leg is in static equilibrium (assuming that the body is fixed). If the reaction forces do not

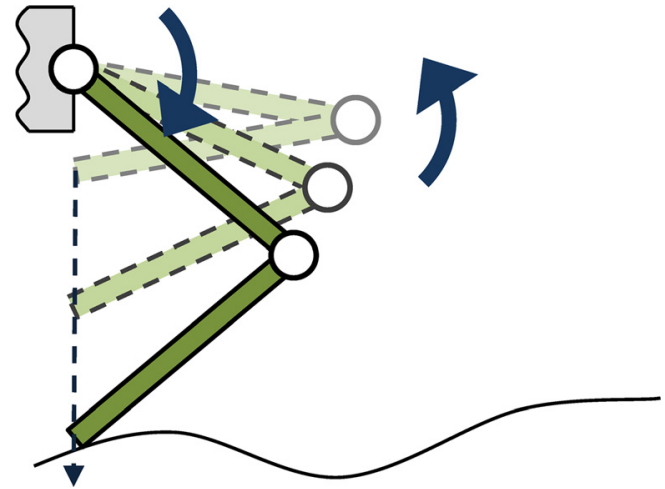

Fig. 8 Representative "push-up" robot leg

match the model, the leg would not be in equilibrium and therefore might be unable to support the robot. The only force opposing $F_{y}$ is the weight of the robot, and this force is constant regardless of leg configuration. Therefore, we desire a leg for which $F_{y}$ is independent of ground height. Similarly, the only force opposing $F_{x}$ is the friction between the foot and the ground. Since this is constrained by the constant normal force, we desire a leg for which minimal horizontal reaction forces are necessary for static equilibrium.

In order to convert those design requirements into numerical quantities, we evaluated each leg design at a number of evenly spaced ground heights and generated force profiles for each. The $F_{y}$ profile for an ideal leg would simply be constant, and the coefficient of variation (CV) was used to evaluate how "constant" the force was over the range of ground heights. Note that the magnitude of $F_{y}$ is not very important as the tendon tension for a given robot design can be set in order to result in the approximate $F_{y}$ value for a given robot weight. The $\mathrm{CV}$ is a normalized measure of the spread of a data set, and is evaluated by dividing the standard deviation of the set by the absolute value of its mean, i.e., $\mathrm{CV}(a)=\sigma(a) /|\mu(a)|$. The $F_{x}$ profile for an ideal leg would be close to zero over the entire range of ground heights, or, more precisely, would be small relative to $F_{y}$ such that it could be supported by friction. This was evaluated using the average of the ratio between the absolute values of the two forces, i.e., $\operatorname{avg}\left(\left|F_{x}\right| /\left|F_{y}\right|\right)$. For both of our force-related performance metrics, lower values mean better performance.

5.2 Simulation Results. Over 300,000 different leg designs were evaluated using the previously defined model. $R_{\mathrm{L}}$ was varied from 0.25 to $1.25, R_{\mathrm{T}}$ was varied from -3 to 3 , and $\theta_{1,0}$ was varied from $-85 \mathrm{deg}$ to $-1 \mathrm{deg}$. A small sample of the force profiles generated by the simulation can be seen in Figs. 9 and 10. The first thing to notice is that the effective range of the legs $\left(y_{\max }\right)$ varies significantly between different designs. In addition, there are a number of $F_{y}$ profiles that, even just by inspection, appear to be fairly constant as well as profiles that are far from constant. Similarly, there are some $F_{x}$ profiles with relatively low magnitudes across the entire range of ground heights as well as profiles with extremely high magnitudes. We will proceed with a more rigorous investigation into the effects of the design parameters on our performance metrics.

\section{Discussion}

6.1 Force Performance Study. Figure 11 shows contours of all three performance metrics (one per column), as a function of $\theta_{1,0}$ (vertical axis) and $R_{\mathrm{T}}$ (horizontal axis), for several values of $R_{\mathrm{L}}$ (rows). First looking at $y_{\max }$, as previously discussed, an balanced leg would reach as far as possible, allowing for the greatest variability in ground height. The first column of Fig. 11 shows a 

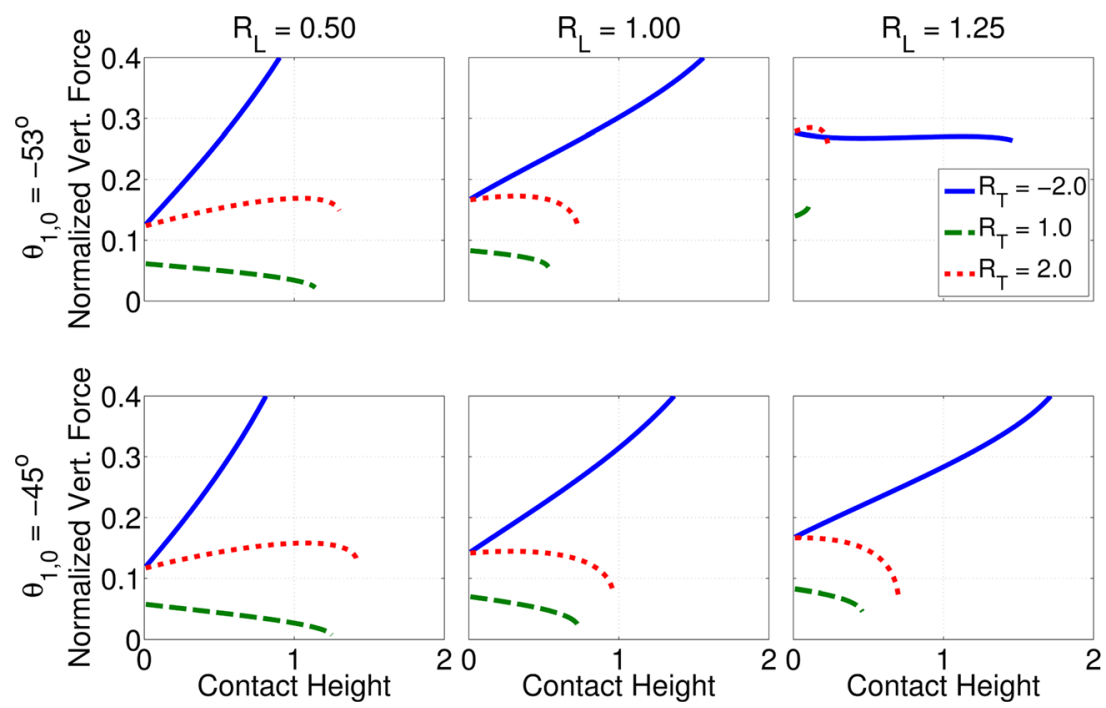

Fig. 9 Comparison of vertical reaction force profiles for a number of different leg designs. Note the change in the overall length of the profiles as well as in their shapes (longer/flatter is better). Each column differs in terms of $\boldsymbol{R}_{\mathrm{L}}$ (link length ratio), while each row differs in terms of $\theta_{1,0}$ (initial joint angle). For each subplot, the force profiles are shown for $R_{\mathrm{T}}$ (joint torque coupling ratio) equal to $-2,1$, and 2 .
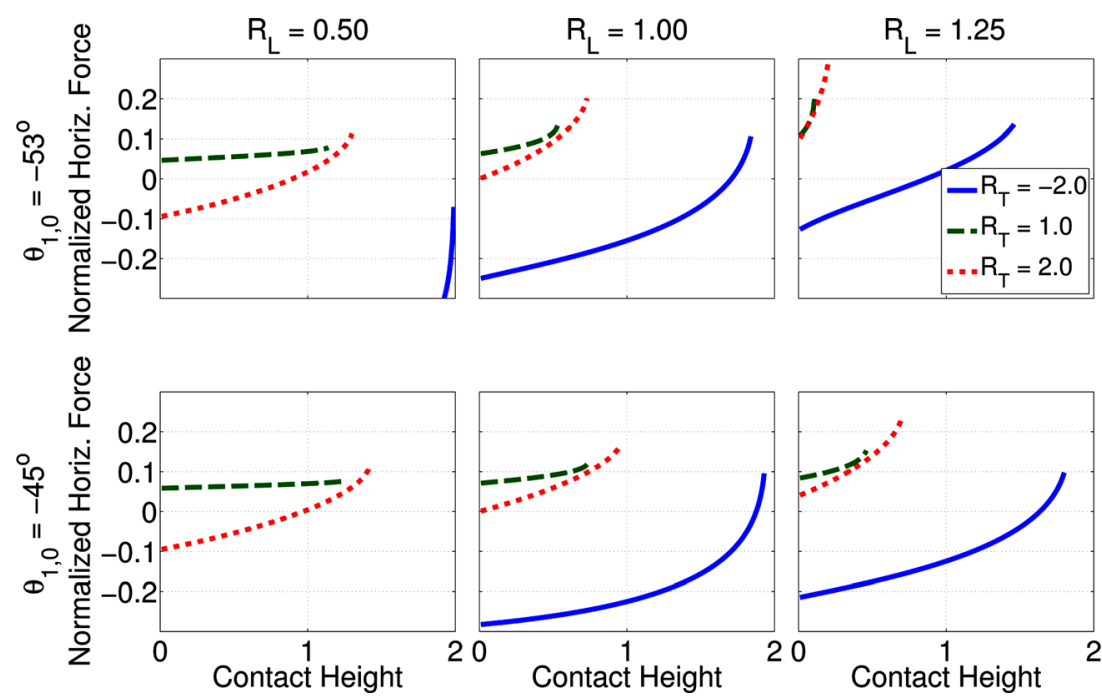

Fig. 10 Comparison of horizontal reaction force profiles for a number of different leg designs. Note the change in the overall length of the profiles as well as in their shapes (longer/centered on zero is better). Each column differs in terms of $R_{\mathrm{L}}$ (link length ratio), while each row differs in terms of $\theta_{1,0}$ (initial joint angle). For each subplot, the force profiles are shown for $\boldsymbol{R}_{\mathrm{T}}$ (joint torque coupling ratio) equal to $-2,1$, and 2 .

series of contour plots of $y_{\max }$ for fixed values of $R_{\mathrm{L}}$, exposing the effect $R_{\mathrm{T}}$ and $\theta_{1,0}$ have on the metric. We notice that for positive values of $R_{\mathrm{T}}$, smaller/less extreme values of $\theta_{1,0}$ result in better performance. This makes sense because the straighter the leg starts out, the longer it can be actuated before the distal segment starts to swing up. There is a region to the left of $R_{\mathrm{T}}=0$ where the legs do not move down at all, after which we see a distinct correlation between the $R_{\mathrm{T}}-\theta_{1,0}$ combination and $y_{\max }$. We note that there is a cross-hatched region for extreme values of $\theta_{1,0}$ on the plots for $R_{\mathrm{L}}=1.25$. This is because legs with $R_{\mathrm{L}}>1$ cannot always reach $y=0$ geometrically; therefore, as $R_{\mathrm{L}}$ increases, the achievable range of $\theta_{1,0}$ gets smaller and smaller (and we cut our parameter space off outside of that range). Finally, we notice that there are basically two regions of good performance on either side of $R_{\mathrm{T}}=0$, one being of the type shown in Fig. $5(a)\left(R_{\mathrm{T}}>0\right)$ and the other being of the type shown in Fig. 7( $a)\left(R_{\mathrm{T}}<0\right)$.

We will now consider the two force-related metrics. The second column of Fig. 11 shows a series of contour plots of $\mathrm{CV}\left(F_{y}\right)$ for fixed values of $R_{\mathrm{L}}$. We see that there is at least one high performance region with respect to $\mathrm{CV}\left(F_{y}\right)$ that moves as $R_{\mathrm{L}}$ increases. For smaller values of $R_{\mathrm{L}}$, this region occupies moderate values of $\theta_{1,0}$ and smaller values of $R_{\mathrm{T}}$. We will refer to this type of leg as positive distal legs. As $R_{\mathrm{L}}$ increases, we see a decrease in performance across that portion of the parameter space with decent performance only occurring at extreme values of $\theta_{1,0}$, which is suboptimal for $y_{\max }$. At the same time, we notice the development of a good performance region appearing for moderately negative values of $R_{\mathrm{T}}$ and more extreme values of $\theta_{1,0}$. This type of leg 

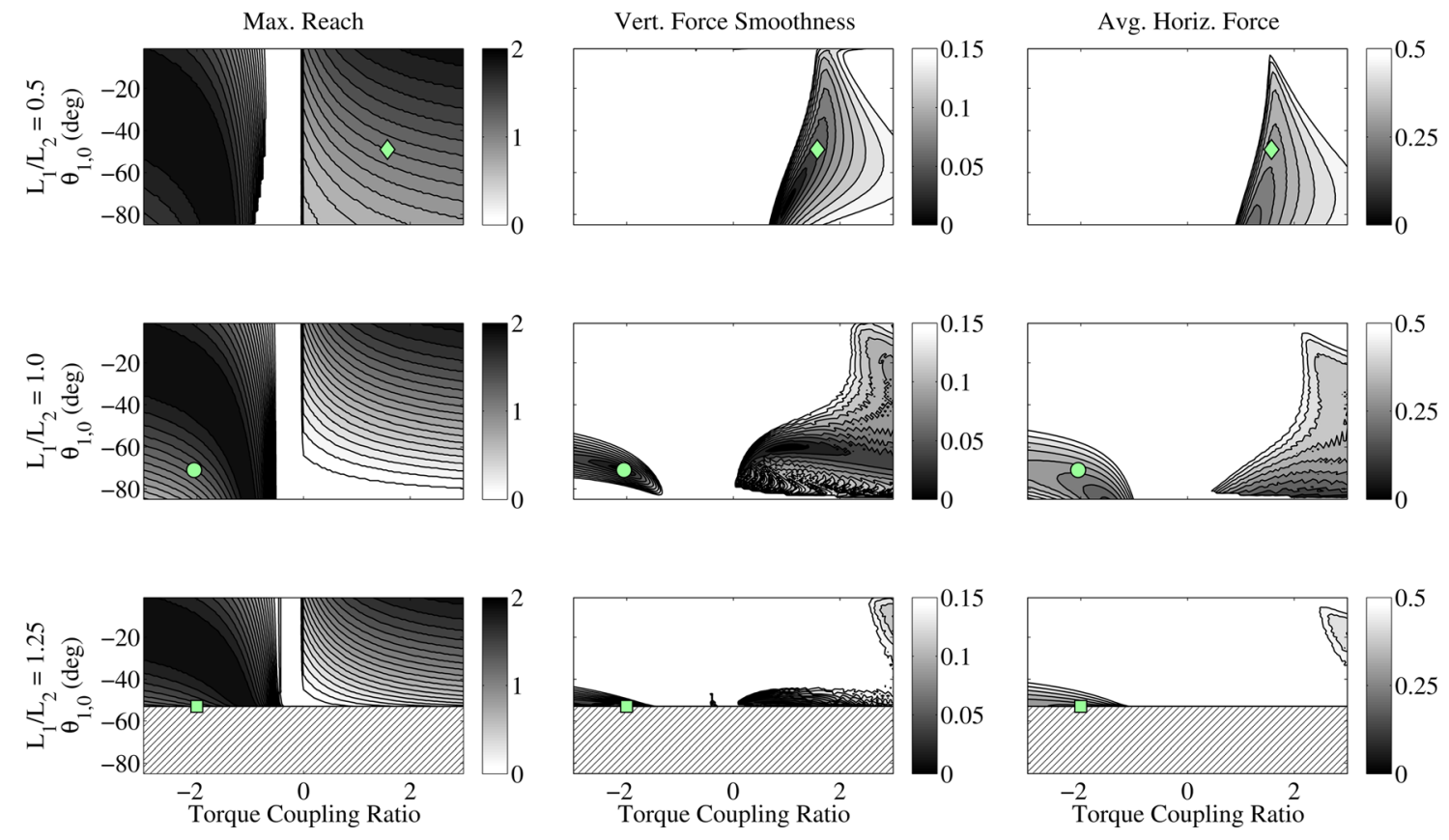

Fig. 11 Contour plots of the three performance metrics, $y_{\max }, \operatorname{CV}\left(F_{y}\right)$, and avg $\left(\left|F_{x}\right| /\left|F_{y}\right|\right)$ for three separate values of $R_{\mathrm{L}}, 0.5,1.0$, and 1.25 (top to bottom). Note the change in behavior as $R_{\mathrm{L}}$ increases. The marker on each row of subplots represents a configuration that balances all three metrics. In all plots, darker regions are preferable.

will be referred to as negative distal legs. As $R_{\mathrm{L}}$ continues to increase, this second region moves to more moderate values of $\theta_{1,0}$ while the first region basically stays the same.

We see a similar pattern in the second force-related performance metric, or $\operatorname{avg}\left(\left|F_{x}\right| /\left|F_{y}\right|\right)$. The third column of Fig. 11 shows a series of contour plots of $\operatorname{avg}\left(\left|F_{x}\right| /\left|F_{y}\right|\right)$ for fixed values of $R_{\mathrm{L}}$. We see that there is at least one preferred performance region for each value of $R_{\mathrm{L}}$ and that the magnitude of the metric tends to increase slightly as $R_{\mathrm{L}}$ increases. The location of the $R_{\mathrm{T}}>0$ preferred performance region changes as $R_{\mathrm{L}}$ changes, but as $R_{\mathrm{L}}$ increases the optimal value of $R_{\mathrm{T}}$ changes more significantly to the point where at $R_{\mathrm{L}}=1$ the optimal $R_{\mathrm{T}}$ is nearly beyond the scope of our parameter space. However, as with $\mathrm{CV}\left(F_{y}\right)$, as $R_{\mathrm{L}}$ passes 1 we see a second preferred performance region develop, basically in the same place as that of the previous metric.

In order to compare all the values of $R_{\mathrm{L}}$, an overall performance metric was developed that addressed the trade-offs between all three metrics. For positive distal legs, it is clear that $R_{\mathrm{L}}=0.5$ gives the best general performance out of the three values displayed in Fig. 11. However, negative distal legs outperformed the positive distal legs across the board, with $R_{\mathrm{L}}=1.25$ giving the most balanced performance out of any other design. For each of the subplots, there is a marker representing the balanced configuration. Figure 12 shows the balanced design at an arbitrary point along its trajectory as well as its force profiles. We see that the leg has decent reach (around 1.5), has a fairly constant $F_{y}$ profile, and that $F_{y}$ is generally much larger than $F_{x}$. Figure 13 shows the representative adaptive legged robot from Fig. 1(b) (modified to match our balanced configuration) with approximate reaction force vectors based on our model. We see that the vertical component of both reaction force vectors is basically constant, as expected, and also that both vectors are primarily vertical, indicating that relatively small frictional forces would be required for static equilibrium (the maximum required friction coefficient is $\mu=0.5$ ). It is reasonable to conclude that this robot would be stable despite the difference in ground height on either side. Rather than concluding our analysis here, we will briefly investigate the effect of the spring constant ratio for completeness.
As mentioned before, the springs serve to return the leg to its default position in the absence of tendon tension. However, the ratio of spring stiffness between the two joints, combined with the

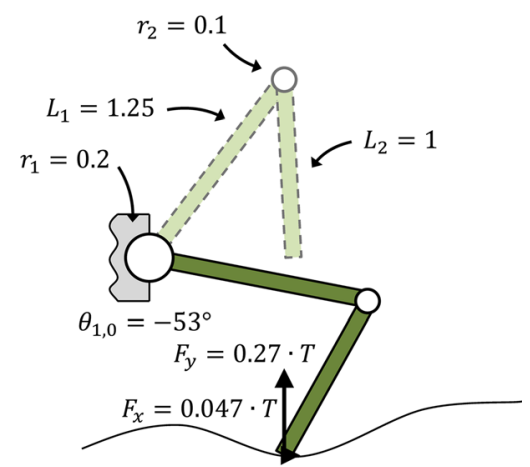

(a)

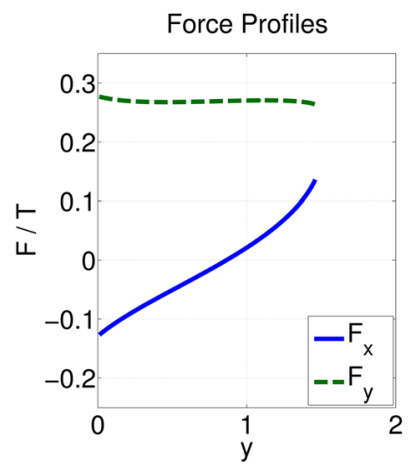

(b)

Fig. 12 (a) Diagram of balanced leg design for $R_{\mathrm{k}}=1$ and (b) reaction force profiles normalized to the tendon tension. On the top, note the normalized dimensions of the leg and the reaction force vectors. On the bottom, note that $F_{y}$ is relatively smooth across the entire range of ground heights and is almost always substantially larger than $F_{X}$. 


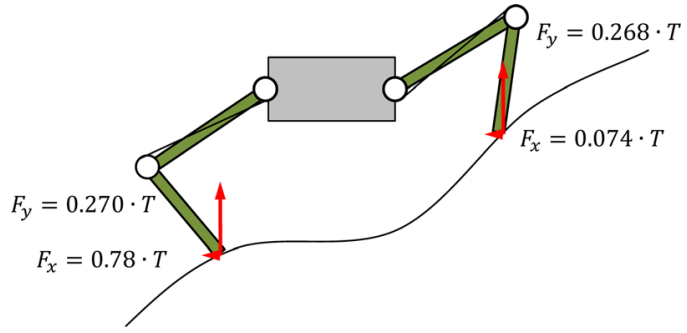

Fig. 13 Diagram of adaptive legged robot standing on rough terrain with approximate reaction force vectors (derived from balanced configuration) drawn and labeled. The ground heights are 0.3 on the right and 1.3 on the left.

torque ratio, also determines the relative motion between the two joints. To put that in mathematical terms, $\Delta \theta_{1} / \Delta \theta_{2}=R_{\mathrm{T}} / R_{\mathrm{k}}$. This is important in determining the configuration of the leg (e.g., the joint angles) when it makes contact at a given ground height, but is otherwise irrelevant. To see if this would impact the performance of the leg, we ran a series of simulations with $R_{\mathrm{k}}=10$, an order of magnitude larger than the results we showed previously.

Figure 14 shows a contour plot for each of our three performance metrics for $R_{\mathrm{k}}=10$ and both $R_{\mathrm{L}}=0.5$ and $R_{\mathrm{L}}=1.25$. For $R_{\mathrm{T}}>0$, we see similar behavior with regard to $\operatorname{avg}\left(\left|F_{x}\right| /\left|F_{y}\right|\right)$ and to a lesser extent with $y_{\max }$, but the behavior of $\mathrm{CV}\left(F_{y}\right)$ is quite different. The preferred region shifts significantly toward moderate values of $\theta_{1,0}$, remaining in the positive distal region. In addition, we see that due to the stiffer proximal joint, our leg cannot move down for the same values of $R_{\mathrm{T}}<0$, pushing our second preferred mode of operation to extreme values of $R_{\mathrm{T}}$. For $R_{\mathrm{L}}=0.5$, if we look at the balanced configuration, we see that the performance metric values for that configuration are similar to those for the balanced $R_{\mathrm{k}}=1 \mathrm{leg}$, with a slight improvements in $\mathrm{CV}\left(F_{y}\right)$.

In order to investigate the effect of $R_{\mathrm{k}}$, we will compare the balanced configurations for $R_{\mathrm{k}}=1$ for both leg types with the balanced configuration for $R_{\mathrm{k}}=10$ and their performance. Table 1 shows both the design parameters for each balanced configuration as well as the values of all three performance metrics for all three legs. We see that the negative distal leg completely outperforms the other two legs according to all three metrics and would require roughly half the actuator force as compared to the positive distal leg for $R_{\mathrm{k}}=1$. The positive distal $R_{\mathrm{k}}=10$ leg slightly outperforms the similar $R_{\mathrm{k}}=1 \mathrm{leg}$, but requires $25 \%$ higher actuator forces to generate equivalent ground reaction forces. From this, we can select the negative distal leg as our optimum.
6.2 Leg Classes Revisited. We will now compare the positive distal leg design and the negative distal leg design. Based on our force performance metrics alone, we concluded that the negative distal leg greatly outperformed the positive distal legs. However, there are other walking issues that can inform the decision between the two classes. Looking at the general trajectory of the two leg types, we see that the robot's foot will be much closer to the body of the robot for the second leg class than for the first. In other words, the positive distal legs would lead to a much more sprawled posture than negative distal legs.

This question therefore boils down to the effect of the overall posture of the robot on its stability. For this discussion, we will introduce a final performance metric - the normalized energy stability margin (NESM) [13], which provides a measure of the resistance of the robot to impact and considers the height of the center of mass. Generally speaking, the NESM is the minimum change in center of gravity height (proportional to change in potential energy) necessary to tip the robot over the hypothetical segment joining the position of two feet. This value for a given segment is defined as follows:

$$
h_{i}=\left|\boldsymbol{R}_{i}\right|(1-\cos \theta) \cos \psi
$$

where $\boldsymbol{R}_{i}$ is the distance between the axis of rotation and the center of mass, $\theta$ is the angle that $\boldsymbol{R}_{\boldsymbol{i}}$ forms with the vertical plane, and $\psi$ is the angle that $\boldsymbol{R}_{\boldsymbol{i}}$ forms with the horizontal plane. In the simplified case, where the feet are inline and the ground is assumed to be horizontal, we can express the height in terms of the foot coordinates $(x, y)$ relative to the center of mass as $h_{i}=\sqrt{x^{2}+y^{2}}-y$.

Figure 15 shows example representations of the NESM for both leg designs. It is immediately clear that the positive distal design will have a much higher NESM than the negative distal design, which largely relies on the body width for the margin. This means that robots using the negative distal leg could be more susceptible to disturbance forces with respect to tip-over and therefore less stable than robots using the positive distal leg. While this is a highly simplified view of walking in unstructured environments, it can nonetheless be useful in designing robots for such a task.

6.3 Limitations and Future Work. There are a number of limitations to this work and a number of things that will be added as this project progresses. One major limitation is that this model only deals with the issue of weight support, ignoring any issues related to actual locomotion (i.e., propulsive force). We envision a design that decouples the propulsion and steering from the stance behaviors. One method of accomplishing this is through a design
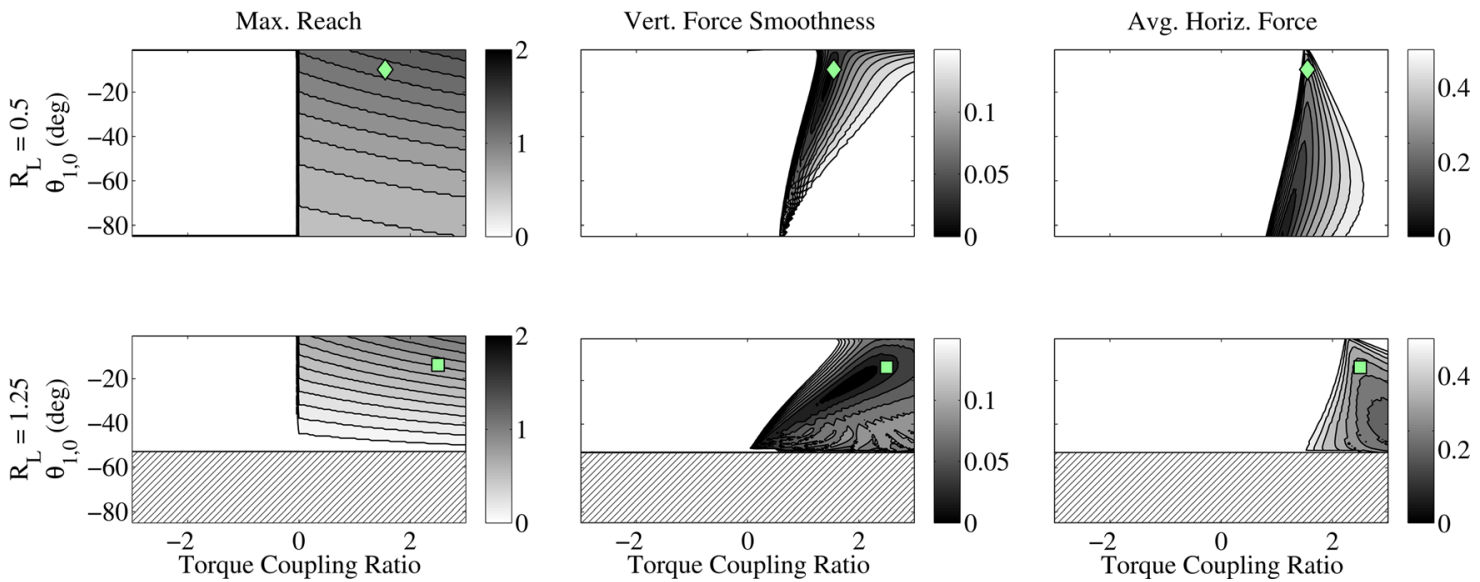

Fig. 14 Contour plots of the three performance metrics, $y_{\max }, \operatorname{CV}\left(F_{y}\right)$, and avg $\left(\left|F_{X}\right| /\left|F_{y}\right|\right)$ for $R_{k}=10\left(R_{L}=0.5\right.$ and $\left.R_{\mathrm{L}}=1.25\right)$. The marker on each subplot represents a configuration that balances all three metrics. Note the performance difference as compared to the $R_{\mathrm{k}}=1$ designs, especially with regards to the balanced performance location and lack thereof for $\boldsymbol{R}_{\mathrm{T}}<0$. 
Table 1 Balanced underactuated leg designs

\begin{tabular}{|c|c|c|c|}
\hline Leg type & Negative distal, $R^{\mathrm{k}}=1$ & Positive distal, $R^{\mathrm{k}}=1$ & Positive distal, $R_{\mathrm{k}}=10$ \\
\hline$R_{\mathrm{L}}$ & 1.25 & 0.5 & 0.5 \\
\hline$R_{\mathrm{T}}$ & -2 & 1.575 & 1.525 \\
\hline$\theta_{1,0}$ & $-53 \mathrm{deg}$ & $-49 \mathrm{deg}$ & $-11 \mathrm{deg}$ \\
\hline Maximum reach & 1.46 & 1.3 & 1.33 \\
\hline Vertical force smoothness & 0.007 & 0.043 & 0.015 \\
\hline Average horizontal force & 0.22 & 0.27 & 0.26 \\
\hline
\end{tabular}

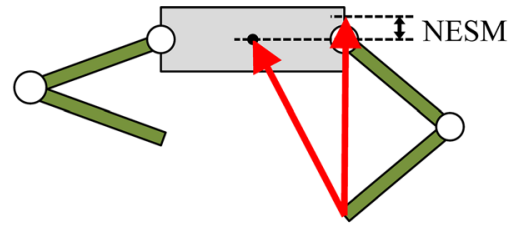

(a)

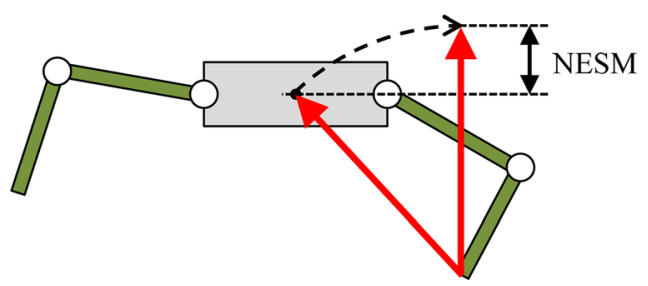

(b)

Fig. 15 Representative robot configurations with NESM shown for (a) positive distal leg design and $(b)$ negative distal leg design with equivalent ground heights

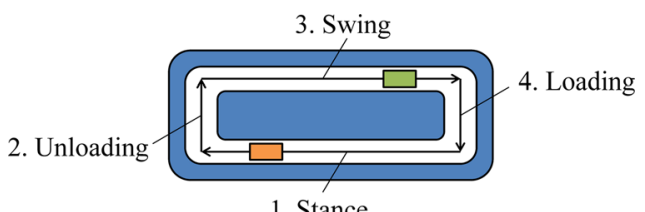

(a)

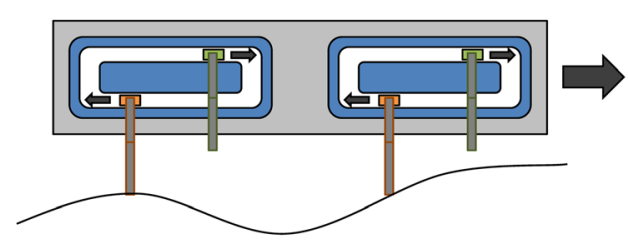

(b)

Fig. 16 Propulsion concept that decouples stance and forward motion. (a) Schematic of propulsion track and the four phases of gait that each leg set (which are $180 \mathrm{deg}$ out of phase) passes through as it cycles and $(b)$ a schematic side view of the legged robot at an instance in time during gait.

that has one stance set "walking over" the other, in a similar way to how tank treads work. In doing so, the stance legs will remain static while the other legs are placed on the ground in front of them. After both sets are in place, the weight of the robot will gradually be taken up by the forward set and removed from the trailing set. In this manner, the legs of the robot will not need to be individually articulated in the direction of motion, which will greatly simplify their design and actuation. Instead, the legs will simply need to be articulated to be brought into contact with the ground and support the mass of the robot. Figure 16 shows a schematic diagram of the proposed propulsion system that decouples stance actuation from propulsion. The four phases of gait are shown in Fig. 16(a), and the leg sets are fully out of phase. As the sets travel, the vertical portions of the track (unloading and loading), the body mass is smoothly transitioned between them. Figure 16(b) shows this track implemented in a pair with one set of the legs in stance and the other in swing.

Looking forward, we hope to enhance our model through the inclusion of the mechanism's "equilibrium point" $[9,14]$ to evaluate potentially unstable configurations. In addition, we will be working toward the development of a physical prototype. In this, we will be implementing between-leg coupling, e.g., actuating multiple legs with a single tendon using a differential transmission, which could vastly improve the stability of such an adaptive robot as well as reduce the required number of actuators. The nature of the specific implementation will be based on a similar, in-depth design study to find coupling configurations that give the best performance.

\section{Acknowledgment}

The authors would like to thank Joseph Belter for his helpful discussions regarding underactuated locomotion and modeling. This work was supported in part by National Science Foundation Grant No. IIS-0952856.

\section{References}

[1] Klaassen, B., Linnemann, R., Spenneberg, D., and Kirchner, F., 2002, "Biomimetic Walking Robot Scorpion: Control and Modeling," Rob. Auton. Syst., 41(2-3), pp. 69-76.

[2] McBride, B., Longoria, R., and Krotkov, E., 2003, "Off-Road Mobility of Small Robotic Ground Vehicles: Measuring the Performance and Intelligence of Systems," Proceedings of the Workshop on Performance Metrics for Intelligent Systems, NIST Special Publication 1014, pp. 405-412.

[3] Raibert, M., Blankespoor, K., Nelson, G., Playter, R., and Team, T. B., 2008, "Bigdog, the Rough-Terrain Quadruped Robot," Proceedings of the International Federation of Automatic Control Congress, pp. 10822-10825.

[4] Birkmeyer, P., Peterson, K., and Fearing, R. S., 2009, "Dash: A Dynamic 16g Hexapedal Robot," Proceedings of the IEEE Intellectual Robots and Systems, pp. $2683-2689$.

[5] Daltorio, K. A., Wei, T. E., Horchler, A. D., Southard, L., Wile, G. D., Quinn, R. D., Gorb, S. N., and Ritzmann, R. E., 2009, "Mini-Whegs TM Climbs Steep Surfaces Using Insect-Inspired Attachment Mechanisms," Int. J. Robot. Res., 28(2), pp. 285-302.

[6] Kim, S., Clark, J. E., and Cutkosky, M. R., 2006, "iSprawl: Design and Tuning for High-Speed Autonomous Open-Loop Running,” Int. J. Robot. Res., 25(9), pp. 903-912.

[7] Saranli, U., Buehler, M., and Koditschek, D. E., 2001, "RHex: A Simple and Highly Mobile Hexapod Robot,” Int. J. Robot. Res., 20(7), pp. 616-631.

[8] Hirose, S., and Umetani, Y., 1978, "The Development of Soft Gripper for the Versatile Robot Hand," Mech. Mach. Theory, 13, pp. 351-359.

[9] Birglen, L., Lalibertçe, T., and Gosselin, C., 2008, Underactuated Robotic Hands, Springer, New York.

[10] Dollar, A. M., and Howe, R. D., 2010, “The Highly Adaptive SDM Hand: Design and Performance Evaluation," Int. J. Robot. Res., 29(5), pp. 585-597.

[11] Inouye, J. M., Kutch, J. J., and Valero-Cuevas, F. J., 2012, "A Novel Synthesis of Computational Approaches Enables Optimization of Grasp Quality of Tendon-Driven Hands,” IEEE Trans. Rob., 28(4), pp. 958-966.

[12] Kanner, O. Y., and Dollar, A. M., 2012, "Optimization of Coupling Ratio and Kinematics of an Underactuated Robot Leg for Passive Terrain Adaptability," Proceedings of the 2012 ASME International Design Engineering Technical Conferences, Mechanisms and Robotics Conference, DETC-70741.

[13] Garcia, E., Estremera, J., and de Santos, P. G., 2002, "A Comparative Study of Stability Margins for Walking Machines," Robotica, 20, pp. 596-606.

[14] Balasubramanian, R., Belter, J. T., and Dollar, A. M., 2010, "External Disturbances and Coupling Mechanisms in Underactuated Hands," Proceedings of the 2010 ASME International Design Engineering Technical Conferences, Mechanisms and Robotics Conference, pp. 175-184. 